\title{
Compact Mach-Zehnder interferometer based on self-collimation of light in a silicon photonic crystal
}

\author{
Hoang M. Nguyen, ${ }^{1, *}$ M. A. Dundar, ${ }^{2}$ R. W. van der Heijden, ${ }^{2}$ E. W. J. M. van der Drift, ${ }^{1}$ \\ H. W. M. Salemink, ${ }^{1}$ S. Rogge ${ }^{1}$ and J. Caro ${ }^{1}$ \\ ${ }^{1}$ Kavli Institute of Nanoscience, Delft University of Technology, Lorentzweg 1, 2628 CJ Delft, The Netherlands \\ ${ }^{2}$ COBRA Inter-University Research Institute, Eindhoven University of Technology, P.O. Box 513, 5600 MB \\ Eindhoven, The Netherlands \\ *m.h.nguyen@tudelft.nl
}

\begin{abstract}
We demonstrate a compact silicon photonic crystal MachZehnder interferometer operating in the self-collimation regime. By tailoring the photonic band structure such as to produce self-collimated beams, it is possible to design beam splitters and mirrors and combine these to a $20 \times 20 \mu \mathrm{m}^{2}$ format. With transmission spectroscopy we find a pronounced unidirectional optical output, the output ratio being as high as 25 at the self-collimation wavelength. Furthermore, the self-collimated beams and the unidirectionality are clearly observed in real space using near-field and far-field optical microscopy. Interpretation of the optical data is strongly supported by different types of simulations.
\end{abstract}

(C)2010 Optical Society of America

OCIS codes: (350.0350) Other areas of optics; (350.4238) Nanophotonics and photonic crystals.

\section{References and links}

1. R. Kirchain, and L. Kimerling, "A roadmap for nanophotonics," Nat. Photonics 1(6), 303-305 (2007).

2. A. Liu, L. Liao, D. Rubin, H. Nguyen, B. Ciftcioglu, Y. Chetrit, N. Izhaky, and M. Paniccia, "High-speed optical modulation based on carrier depletion in a silicon waveguide," Opt. Express 15(2), 660-668 (2007).

3. S. P. Anderson, A. R. Schroff, and P. M. Fauchet, "Slow light with photonic crystal for on-chip optical interconnects," Advances in Optical Technologies (2008).

4. Y. A. Vlasov, M. O'Boyle, H. F. Hamann, and S. J. McNab, "Active control of slow light on a chip with photonic crystal waveguides," Nature 438(7064), 65-69 (2005).

5. M. A. Mansouri-Birjandi, M. K. Moravvej-Farshi, and A. Rostami, "Ultrafast low-threshold all-optical switch implemented by arrays of ring resonators coupled to a Mach-Zehnder interferometer arm: based on 2D photonic crystals," Appl. Opt. 47(27), 5041-5050 (2008).

6. H. Kosaka, T. Kawashima, A. Tomita, M. Notomi, T. Tamamura, T. Sato, and S. Kawakami, "Self-collimating phenomena in photonic crystals," Appl. Phys. Lett. 74(9), 1212-1215 (1999).

7. J. Witzens, M. Loncar, and A. Scherer, "Self-collimation in planar photonic crystals," IEEE J. Sel. Quantum Electron. 8(6), 1246-1257 (2002).

8. P. T. Rakich, M. S. Dahlem, S. Tandon, M. Ibanescu, M. Soljačić, G. S. Petrich, J. D. Joannopoulos, L. A. Kolodziejski, and E. P. Ippen, "Achieving centimetre-scale supercollimation in a large-area two-dimensional photonic crystal," Nat. Mater. 5(2), 93-96 (2006).

9. J. D. Joannopoulous, S. G. Johnson, J. N. Winn, and R. D. Meade, Photonic crystal: Molding the flow of light (Princeton University Press, 2008).

10. D. W. Prather, S. Shi, D. M. Pustai, C. Chen, S. Venkataraman, A. Sharkawy, G. J. Schneider, and J. Murakowski, "Dispersion-based optical routing in photonic crystals," Opt. Lett. 29(1), 50-52 (2004).

11. DD. W. Prather, S. Shi, J. Murakowski, G. J. Schneider, A. Sharkawy, C. Chen, B. L. Miao, and R. Martin, "Self-collimation in photonic crystal structures: a new paradigm for applications and device development," J. Phys. D Appl. Phys. 40(9), 2635-2651 (2007).

12. X. Yu, and S. Fan, "Bends and splitters for selft-collimated beams in photonic crystals," Appl. Phys. Lett. 83(16), 3251-3253 (2003).

13. S.-G. Lee, S. S. Oh, J.-E. Kim, H. Y. Park, and C.-S. Kee, "Line defect induced bending and splitting of selfcollimated beams in two-dimentional photonic crystals," Appl. Phys. Lett. 87, 118106 (2005).

14. D. Zhao, J. Zhang, P. Yao, X. Jiang, and X. Chen, "Photonic crystal Mach-Zehnder interferometer based on selfcollimation," Appl. Phys. Lett. 90, 231114-1-231114-3 (2007)

15. Finite-element frequency-domain simulations are done using the Finite Element Frequency Domain (FEFD) engine from Photon Design. http://www.photond.com/products/fefd. The FEFD engine is a 2D Maxwell solver 
for propagation of electromagnetic fields within an arbitrary photonic structure, which allows one to compute a steady state response for a single frequency. For further reference see [16].

16. T. P. Felici, D. F. G. Gallagher, and L. Bolla, "Automatic Design and Optimisation of Si nanophotonics devices using Finite Element Frequency Domain Solvers," in Proceedings of SPIE Vol. 6475, Integrated Optics: Devices, Materials, and Technologies XI(2007), pp. 64750L-1-64750L-9.

17. R. Ramaswami, and K. N. Sivarajan, Optical networks: A pratical perspective (Morgan Kaufmann, San Francisco, 1998).

18. L. Z. Zehnder, "Ein neuer Interferenzrefraktor," Instrumentenkunde 11, 275-285 (1891).

19. L. Z. Mach, "Ueber einen Interferenzrefraktor,” Instrumentenkunde 12, 89-94 (1892).

20. J. Caro, E. M. Roeling, B. Rong, H. M. Nguyen, E. W. J. M. van der Drift, S. Rogge, F. Karouta, R. W. van der Heijden, and H. W. M. Salemink, "Transmission measurement of the photonic band gap of GaN photonic crystal slabs," Appl. Phys. Lett. 93(5), 051117-051119 (2008).

21. K. Vynck, E. Centeno, M. L. Vassor d'Yerville, and D. Cassagne, "Efficient light coupling from integrated single-mode waveguides to supercollimating photonic crystals on silicon-on-insulator platforms," Appl. Phys. Lett. 92(10), 103128-1, 103128-3 (2008).

22. S.-G. Lee, J. S. Choi, J.-E. Kim, H. Y. Park, and C.-S. Kee, "Reflection minimization at two-dimensional photonic crystal interfaces," Opt. Express 16(6), 4270-4277 (2008).

23. I. Horcas, R. Fernández, J. M. Gómez -Rodriguez, J. Colchero, J. Gómez -Herrero, and A. M. Baro, "WSXM: A software for scanning probe microscopy and a tool for nanotechnology," Rev. Sci. Instrum. 78(1), 013705-1, 013705-013708 (2007).

24. W. Zhou, D. M. Mackie, M. Taysing-Lara, G. Dang, P. G. Newman, and S. Svensson, "Novel reconfigurable semiconductor photonic crystal-MEMS device," Solid-State Electron. 50(6), 908-913 (2006).

25. K. L. Ekinci, and M. L. Roukes, "Nanoelectromechanical systems," Rev. Sci. Instrum. 76(6), 061101-1, 061101-061112(2005).

26. T. Takahata, K. Hoshino, K. Matsumoto, and I. Shimoyama, in Proceedings of MEMS (Instanbul, Turkey, 2006), pp. 834-837.

\section{Introduction}

Optical waveguides based on silicon-on-insulator (SOI) and fabricated in CMOS compatible technology will likely replace electrical interconnects for high data rate circuits [1-3]. In this context, a modulator based on a silicon-waveguide Mach-Zehnder interferometer (MZI) is very promising [2]. However, the requirement of producing a $\pi$ phase shift in one arm of the MZI implies a millimeter scale footprint of the device, which is a serious disadvantage for integration in micro-processors [3]. Therefore, strong efforts are made to miniaturize MZI modulators without compromising the required phase shift, particularly using slow light engineering in photonic crystals (PhCs) [3-5]. Here, we demonstrate a different concept for a compact MZI built from a PhC operating in the special regime of self-collimation of light. To reach this regime, we have tailored the photonic band structure such as to produce selfcollimated beams. The properties of these beams are such that we can manipulate them to form the arms of the MZI in a very small area of $20 \times 20 \mu \mathrm{m}^{2}$. Our approach holds a promise for a compact MZI modulator.

Self-collimation of light in a $\mathrm{PhC}$ is a unique phenomenon, characterised by light propagation in particular directions without diffraction [6-8]. It originates from the possiblity to partly convert, in a specially designed $\mathrm{PhC}$, the circular equi-frequency contours (EFCs) of free space into highly anisotropic EFCs with flat sections. For each point ( $\vec{k}$-vector) on such a section the group velocity $\vec{v}_{\vec{k}}=\partial \omega / \partial \vec{k}$ points in the same direction. Therefore, diffractioninduced admixture of $k$-vectors adjacent to an initial k-vector located on a flat section and defining the initial propagation direction of a beam, will almost not affect the beam width [9]. In other words: classical diffraction as a mechanism of beam spreading is counteracted by the intrinsic properties of the photonic band structure of the PhC. Self-collimation can be made functional in several ways, the most evident one being self-guiding of light $[8,10,11]$, which makes fabricated waveguides superfluous. Although beam splitters and mirrors inside a PhC operating in the self-collimation mode have been realized and studied as separate elements [11-13], these photonic building blocks have not yet been combined in an operational $\mathrm{PhC}$ MZI.

\#121075 - \$15.00 USD Received 7 Dec 2009; revised 15 Jan 2010; accepted 16 Feb 2010; published 15 Mar 2010 


\section{MZI design in the self-collimation regime}

The PhC MZI reported here has been designed for a $220 \mathrm{~nm}$ thick device layer of SOI material (SOI material: the $\mathrm{Si}$ device layer is on top of a $\mathrm{SiO}_{2}$ layer, which separates the device layer from the substrate). In particular, we choose hole-type $\mathrm{PhC}$ slabs with a square lattice. The reasons are that hole slabs provide stronger vertical mode confinement than the pillar slabs in the MZI simulations by Zhao et al. [14] and that the square lattice, in view of the topology of its EFCs, is the obvious choice for the self-collimation effect and for the MZI geometry.

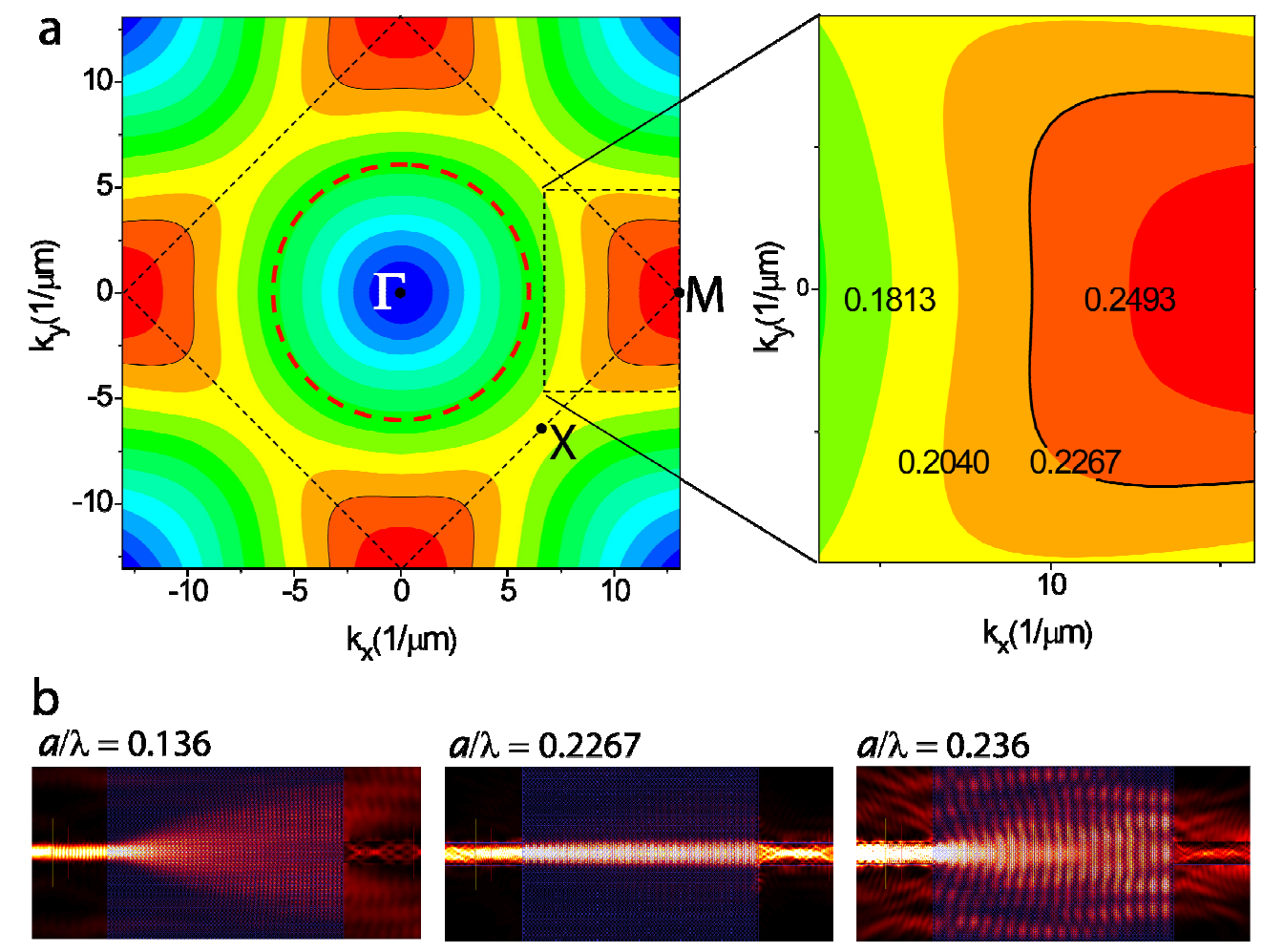

Fig. 1. (a) Equi-frequency contours (EFCs) in the first two Brillouin zones for the lowest TE photonic band of the square lattice PhC. The colours indicate regions between EFCs, each characterized by a normalized frequency $a / \lambda$. In the blue and green regions the dispersion is isotropic, i.e. here the TE modes of the $\mathrm{PhC}$ undergo classic diffraction, as in a homogeneous isotropic medium. The blow-up of the region at the M-point shows highly anisotropic yellow, orange and red regions ( $a / \lambda$ between 0.1813 and 0.2493 ). The flat section labeled with $a / \lambda$

$=0.2267$ is responsible for the self-collimated beams excited at $\lambda=1.50 \mu \mathrm{m}$. The dashed red circle is the cross section of the light cone for $\mathrm{SiO}_{2}$ with the equi-frequency plane at $a / \lambda_{c}=$ 0.2267. (b) Simulations of the propagation of TE modes through the PhC between collinear input and output waveguides, for $a / \lambda=0.136, a / \lambda_{c}=0.2267$ and $a / \lambda=0.236$, respectively.

Using CrystalWave, a commercial software package of Photon Design, we calculate the photonic band structure for $a=340 \mathrm{~nm}$ and $r=105 \mathrm{~nm}(r$ is hole radius and $a$ is lattice constant). These $a$ and $r$ values yield optimum self-collimation for modes in the $\Gamma \mathrm{M}$ branch of the lowest transverse electric (TE) band that can be excited with an external laser operating at the self-collimation wavelength $\lambda_{c}=1.50 \mu \mathrm{m}$. The refractive indices used in this calculation are $n_{\mathrm{Si}}=3.5$ and $\mathrm{n}_{\mathrm{SiO}_{2}}=1.46$. The result is shown in Fig. 1(a), in the form of 
EFCs in the first two Brillouin zones. The crucial features are the flat sections of the EFC at $a / \lambda_{c}=0.2267$ (black curves in Fig. 1(a)) facing the $\Gamma$ point. These sections and similar ones in their immediate vicinity (not shown) give rise to the self-collimated beams which are at the basis of the MZI we study. These self-collimation modes are strongly confined inside the silicon slab and thus cannot scatter into the $\mathrm{SiO}_{2}$ layer, as guaranteed by the fact that their EFCs are far outside the light cone. This can be seen in Fig. 1(a), where the dashed red circle is the cross section of the light cone for $\mathrm{SiO}_{2}$ with the equi-frequency plane at $a / \lambda_{c}=0.2267$. In Fig. 1(b), a self-collimated beam with its constant width can be distinguished clearly from non-collimated beams with their typical diffraction-induced broadening.

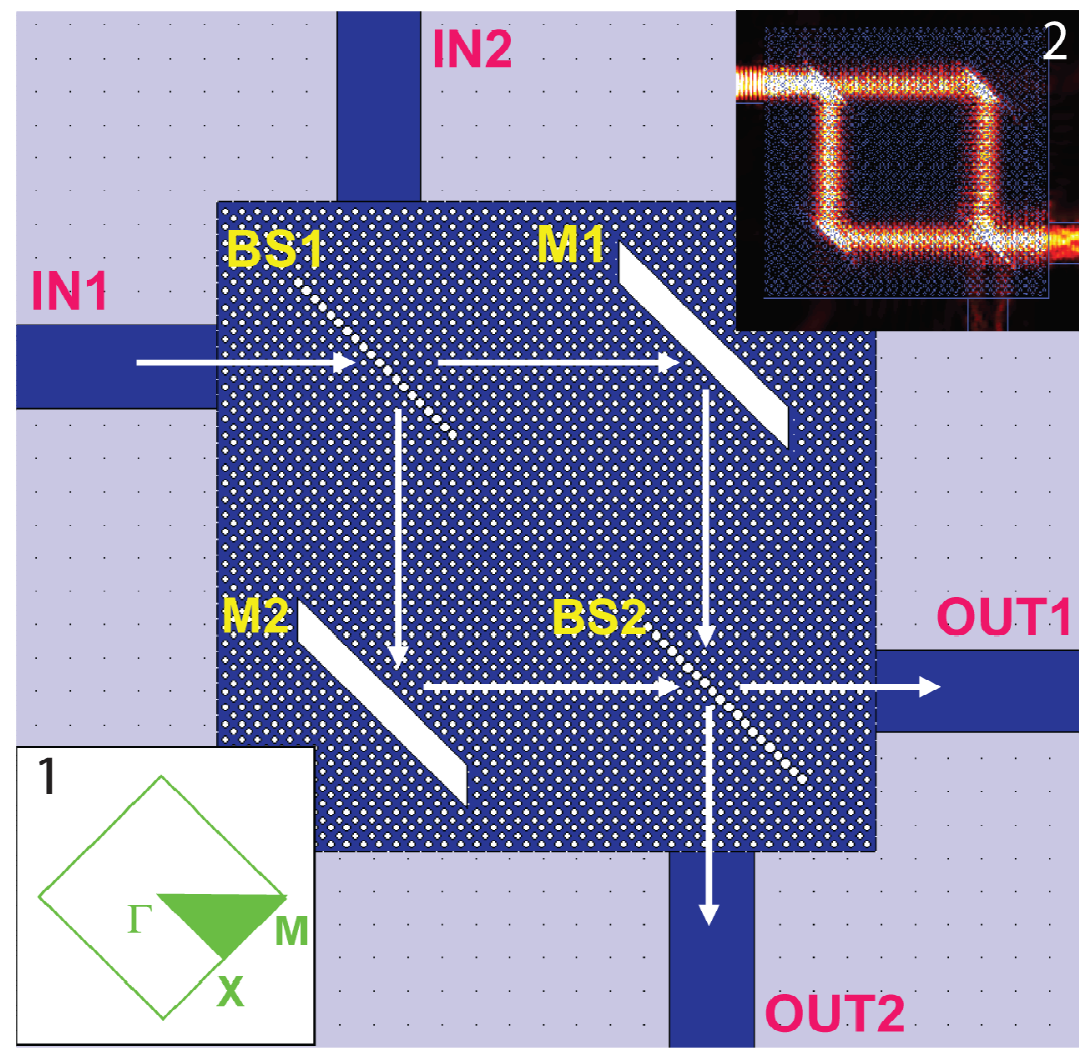

Fig. 2. Design of the PhC MZI. BS1 and BS2 are beam splitters, defined by a line defect. M1 and $\mathrm{M} 2$ are mirrors, defined by air regions. IN1, IN2 and OUT1, OUT2 are input and output waveguides, respectively, for light coupling. White arrows indicate the direction of light propagation. Inset 1 shows the first Brillouin zone (BZ), with in green the irreducible BZ, which defines the symmetry directions $\Gamma M$ and $\Gamma X$ in the PhC. Inset 2 is a $2 \mathrm{D}$ FEFD simulation result at the collimation wavelength, showing the unidirectional output behaviour of the PhC MZI. Details of the design are given in the text.

The beam splitters BS1 and BS2 of the symmetric MZI are each designed as a line defect, viz. a single row of air holes larger $\left(r_{B S}=155 \mathrm{~nm}\right)$ than those of the regular PhC. See Fig. 2 for the position of BS1 and BS2 in the overall geometry of the MZI and for the light paths in its two arms. The total internal reflection mirrors M1 and M2 are parallelogram-shaped air regions, three rows of air holes wide and with smooth edges. The components BS1, BS2 and M1, M2 are oriented parallel along the same $\Gamma \mathrm{X}$ direction and centered at the corners of a square with side $20 a \sqrt{2}=9.6 \mu \mathrm{m}$, which results in a compact total size of the PhC MZI of 20 $\times 20 \mu \mathrm{m}^{2}$. The details of the beam splitters and mirrors are separately fine-tuned with $3 \mathrm{D}$ finite-difference time-domain (FDTD) simulations, to obtain at the self-collimation 
wavelength a splitting characteristic close to the optimum 50\%/50\% and a reflection characteristic close to the optimum $100 \%$, respectively. Further details of the MZI include input waveguides IN1, IN2 and output waveguides OUT1, OUT2, each $2.5 \mu \mathrm{m}$ wide (see Fig. 2).

By tracing the light paths in the MZI and taking into account the phase shifts occurring at the beam splitters and mirrors, it follows that the beams recombining at OUT1 are in phase and thus interfere constructively (maximum intensity), while the beams recombining at OUT2 are out of phase and thus interfere destructively (zero intensity). Inset 2 of Fig. 2 clearly demonstrates this unidirectional output behaviour, as obtained from 2D finite-element frequency-domain (FEFD) simulations $[15,16]$ at the self-collimation wavelength. The unidirectionality is similar as occurring in the symmetric integrated-optics MZI with waveguide couplers [17] and in the classical MZI with symmetric arms [18,19]. The latter, owing to the sensitivity of the ratio of the output signals to a phase shift introduced in one of the arms, has been widely applied as optical sensing instrument of macroscopic samples. The same sensitivity of the outputs to a phase shift in an arm may be used to make the PhC MZI tunable.

\section{Device fabrication}

The main fabrication steps of the MZI are electron-beam lithography and cryogenic inductively coupled plasma (ICP) etching. A $120 \mathrm{~nm}$ thick ZEP resist layer is directly spincoated onto the SOI chip. For patterns writing we use a Leica 5000 + electron-beam pattern generator. The complete MZI pattern is written in a single writing step. After resist development, the pattern is transferred to the silicon device layer using ICP etching in a $\mathrm{SF}_{6} / \mathrm{O}_{2}$ plasma at a substrate temperature of $-120^{\circ} \mathrm{C}$. The remaining resist is then stripped in PRS3000 at $75^{\circ} \mathrm{C}$ for 1 hour. Finally, the sample is cleaved perpendicularly through the waveguides to form waveguide facets.

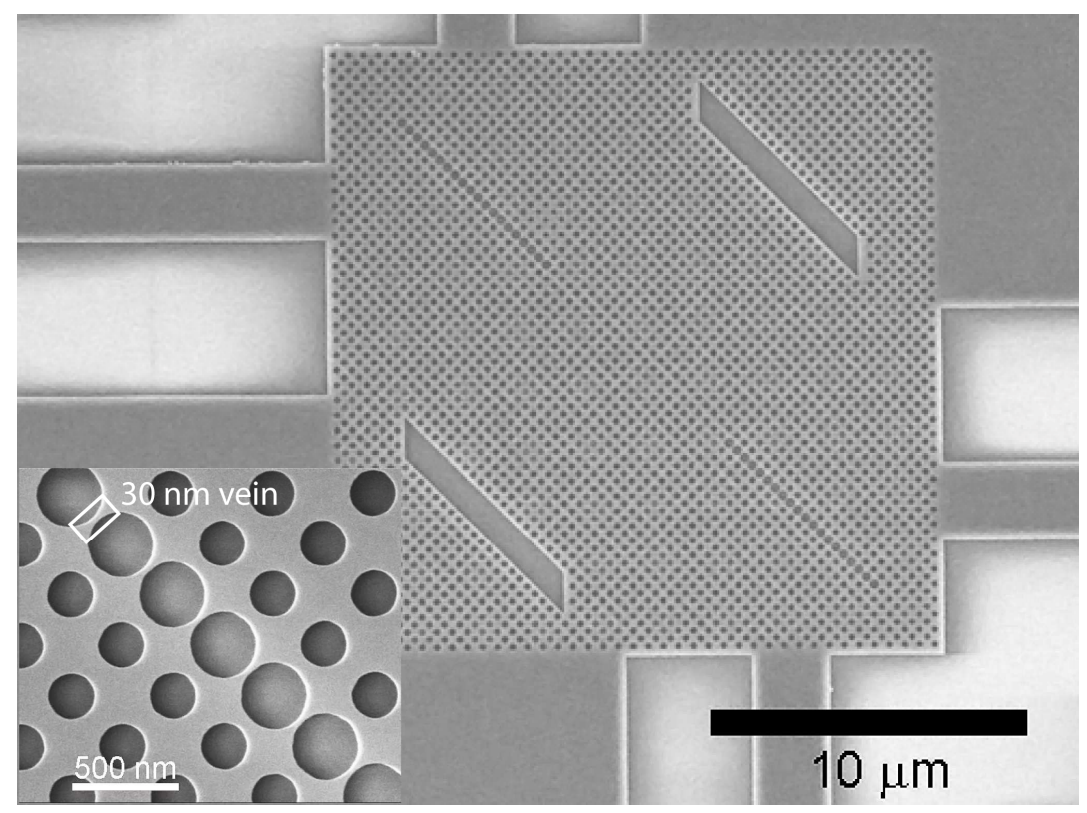

Fig. 3. SEM image of the PhC MZI, demonstrating accurate realization of the design. All structures ( $\mathrm{PhC}$ holes, beam splitters, mirrors and waveguides) are etched in a single etching step. The inset is an SEM image of a beam splitter, featuring smooth circular holes. The hole radius for the regular $\mathrm{PhC}$ is $r \approx 105 \mathrm{~nm}$, while for the line defect it is $r_{B S} \approx 155 \mathrm{~nm}$, consistent with the design. A narrow vein of a beam splitter is highlighted in the white rectangle. 
In Fig. 3 we show a scanning electron microscope (SEM) image of a fabricated MZI. We emphasize, on the basis of our experimental observation of the strong sensitivity of the MZI performance to its geometrical details, the importance of accurate dimensional control of the circular air holes and of their homogeneity across the PhC. In view of this we apply a proximity-effect correction in electron-beam writing of the lithographic patterns. Further, the side wall of each type of etched feature should be smooth and vertical. High fabrication demands in particular apply to the beam splitters and the mirrors. Any small deviation from the design of the beam splitters leads to a decay of the optical performance. For instance, a beam splitter would present a wrong phase shift, optical loss, unequal splitting or even mirrorlike operation if its narrow veins (30 nm; see inset of Fig. 3) would be unintentionally narrower or removed. However, the high quality measurements data, discussed in the next section, indicate that we meet the above fabrication challenges with our simple, but dedicated fabrication process.

\section{Measurements and interpretation}

\section{Transmission measurement}

In Fig. 4 we present optical transmission measurements obtained with the end-fire technique [20]. The measurements are driven by a tunable near-infrared laser (Santec TSL-210VF, 1.44$1.63 \mu \mathrm{m})$. Waveguide facets are used for in- and out-coupling of light and a spatial filter with a $25 \mu \mathrm{m}$ diameter pinhole is used to filter out stray light. The transmitted light is measured with an InGaAs photo-detector. Finally, a lock-in technique is used for noise reduction. The signal at OUT1 is peak-shaped and shows a maximum very close to the designed selfcollimation wavelength $\left(\lambda_{c}=1.50 \mu \mathrm{m}\right)$, while the signal at OUT2 is on average 15 times lower and even 25 times lower at the self-collimation wavelength. This is the expected unidirectional output behaviour of the MZI and can only be understood in terms of selfcollimated beams in the arms of the MZI. In more detail, the spectrum at OUT1 results from:

i) the spectrum of TE-polarized modes injected into the PhC MZI from IN1 (depending on the mode coupling efficiency between the waveguide and the $\mathrm{PhC}$ ),

ii) spreading of the beams when $\lambda$ deviates from the self-collimation wavelength $\lambda_{c}$ (see Fig. 1(b); this effect gives signal decrease when $\lambda$ is tuned away from $\lambda_{c}$, since then not all the light is collected),

iii) the wavelength dependence of the beam splitter properties (also giving signal decrease when $\lambda$ is tuned away from $\lambda_{c}$ ).

The net effect of the above ingredients is observed as a wavelength dependent interference result after BS2, at OUT1 and OUT2. The data plotted in Fig. 4 were smoothed to take out the high-order Fabry-Perot fringes arising from the cavities delimited between the facet of waveguides IN1, OUT1, OUT2 and the waveguide/PhC interfaces [20] and the low-order Fabry-Perot fringes, mainly arising from the shorter cavities delimited between the waveguide/PhC interfaces. These fringes can be suppressed by improving the mode-matching between waveguide and $\mathrm{PhC}$, for example by using rib waveguides instead of ridge waveguides [21], or by entering the light into the $\mathrm{PhC}$ with a free-space beam and using an antireflection coating structure [22]. In future work, we will include mode-matching structures in order to improve the optical performance of the PhC MZI. The inset of Fig. 4 shows the unsmoothed experimental data, with the main characteristics being hidden by the Fabry-Perot fringes.

\#121075 - \$15.00 USD Received 7 Dec 2009; revised 15 Jan 2010; accepted 16 Feb 2010; published 15 Mar 2010 


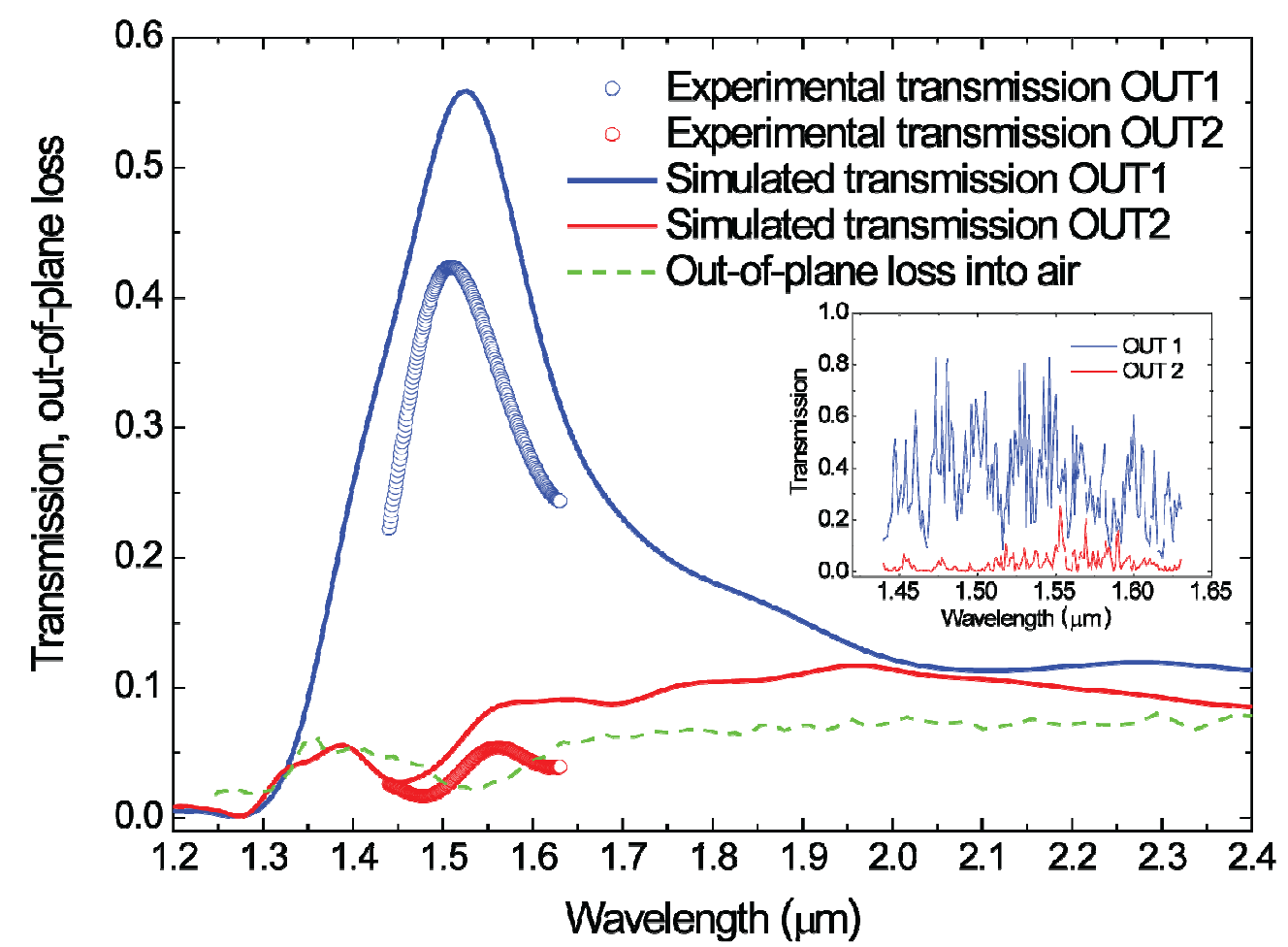

Fig. 4. Experimental and simulated transmission spectra of the MZI and simulated out-of plane loss into the air cladding of the MZI (see legend in panel). Both the experimental and the simulated transmission spectra have been normalized with ridge waveguides. Inset shows the unsmoothed data with the Fabry-Perot fringes.

Interpretation of the optical data as arising from self-collimation is supported by 3D FDTD simulations of the spectra at OUT1 and OUT2, in Fig. 4 plotted as continuous curves in the wavelength range 1.2-2.4 $\mu \mathrm{m}$. In these curves, the low-order Fabry-Perot fringes as present in the raw simulation data have been filtered out, since we concentrate on the unidirectional output behaviour. Measurements and simulations of the spectra agree both for the global shape of the signals and for their magnitude. Outside the experimental wavelength range, on either side, the simulated spectrum for OUT1 continues to decay, due to the same effects causing the decay of the experimental signal. At the long wavelength side the simulated transmission subsequently saturates at a value of about 0.1 , approximately equal to the transmission for OUT2. Far away from the self-collimation wavelength, the interference conditions for the beams recombining at BS2 apparently are such that the distinction between the two outputs is lost. At $\lambda_{c}$ the simulated transmission at OUT2 clearly has its lowest value, just like in the experimental curve. Eventually, with decreasing $\lambda\left(\lambda<\lambda_{c}\right)$, the transmissions at OUT1 and OUT2 virtually vanish at $\lambda \approx 1.3 \mu \mathrm{m}$, where the $\Gamma \mathrm{M}$ stop gap of the PhC is entered.

\section{Near-field measurements}

Furthermore, the MZI operation in and out of the self-collimation condition (1.51 and 1.62 $\mu \mathrm{m}$, respectively) has been observed in real space by measuring the evanescent field of the inplane modes (see Fig. 5) using a scanning near-field optical microscope (SNOM) system (MultiView $4000^{\mathrm{TM}}$ ) from Nanonics Imaging Ltd.. Tapered fibers (spot diameter $1.7 \pm 0.3$ $\mu \mathrm{m})$ are used for in- and out-coupling of light at the sample's waveguide facets. The evanescent field is scanned with a $500 \mathrm{~nm}$ diameter SNOM tip (metal coated). 

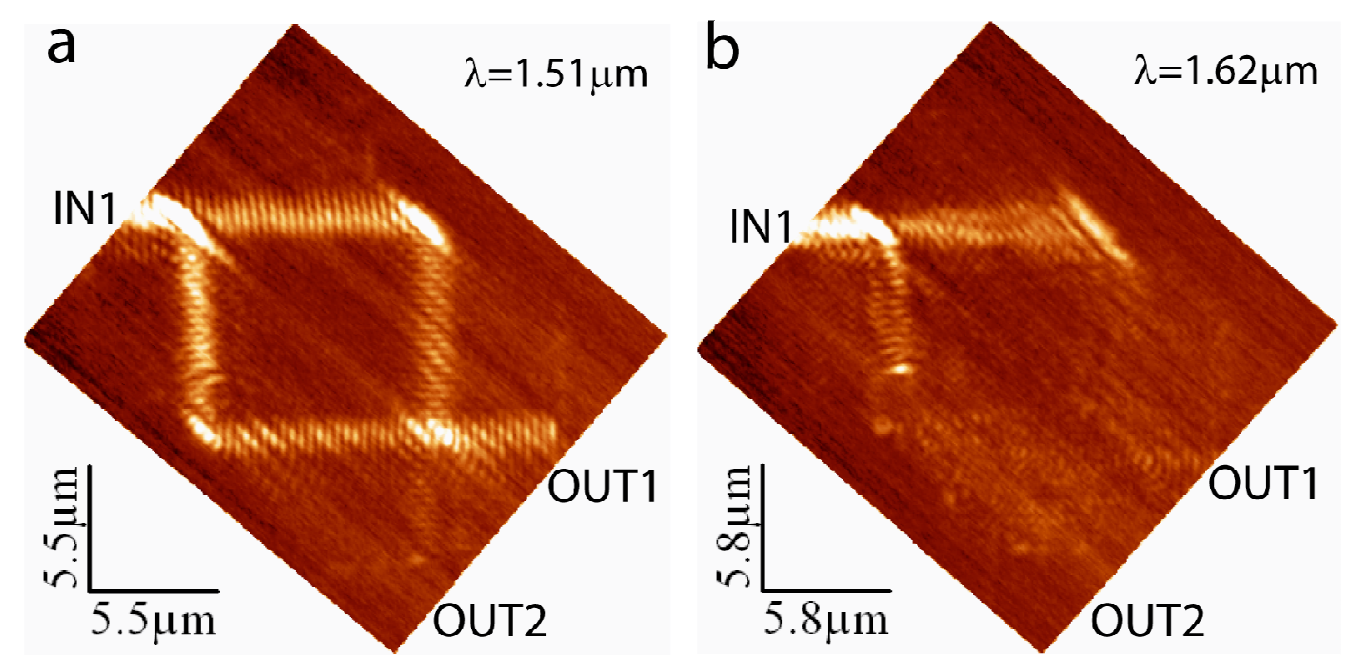

Fig. 5. (a), (b) NSOM images of the MZI for on-collimation operation $(\lambda=1.51 \mu \mathrm{m})$ and offcollimation operation $(\lambda=1.62 \mu \mathrm{m})$, respectively. The unidirectional output behaviour is clearly seen in the on-collimation case, but is lost in the off-collimation case.

The SNOM image for the on-collimation case was obtained at $1.51 \mu \mathrm{m}$, the minimum wavelength of the laser source attached to the SNOM set-up. Figure 5(a) shows the near-field intensity of the collimated beams propagating through the MZI and in addition illustrates the unidirectional output behaviour as obviously as the simulation in the inset 2 of Fig. 2. When the wavelength is tuned away from the self-collimation condition, the unidirectionality is lost as a consequence of the beam spreading and the beam splitters' wavelength dependence (see Fig. 5(b). With these SNOM images (post-processed using WSxM 4.0 [23]), we directly prove the unidirectionality of the PhC MZI operating in the self-collimation mode.

\section{Far-field measurement}

In the experimental data and simulations the total transmission at the self-collimation wavelength, given by the addition of the signals at OUT1 and OUT2, amounts to only about 0.5-0.6 (see Fig. 4) instead of the expected value of unity. To establish whether out-of-plane optical loss is involved here, we imaged the leakage of in-plane modes to extended modes propagating in air using an infrared microscope set-up, both under the exact self-collimation condition $\left(\lambda_{c}\right)$ and away from that $(\lambda=1.62 \mu \mathrm{m})$. The top-view observations of out-of-plane optical loss are made with a Navitar Zoom lens microscope with a 17-mm working distance Nikon 50x objective and a Xenics infrared camera (XEVA, $0.9-1.7 \mu \mathrm{m})$. 

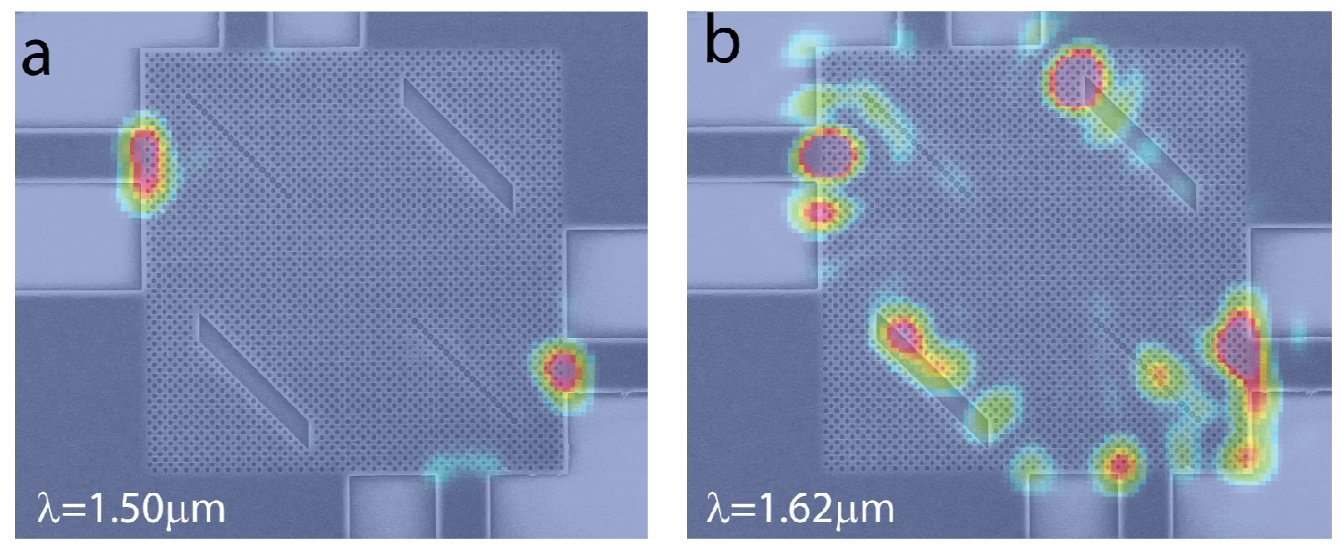

Fig. 6. (a), (b) Top-view optical microscopic images of the MZI for on-collimation operation $\left(\lambda_{c}=1.50 \mu \mathrm{m}\right)$ and off-collimation operation $(\lambda=1.62 \mu \mathrm{m})$, respectively, with a SEM image of the MZI superimposed. As seen from the number of hot spots, out-of plane losses are much weaker for the on-collimation case than for the off-collimation case.

In Figs. 6(a) and 6(b) the resulting images are shown. These give no sign of out-of-plane loss of the in-plane modes propagating between the beam splitters and mirrors, in agreement with the nature of these strongly confined modes and confirming the high structural quality of our hole-type PhC. On collimation, two strong hot spots are seen at the interface between the $\mathrm{PhC}$ and the waveguides IN1 and OUT1 (see Fig. 6(a)). We interpret the hot spots as the result of mode-mismatching at these interfaces. This mode-mismatch causes reflections and out-of plane losses into the $\mathrm{SiO}_{2}$ cladding and air cladding, the latter loss being observed here. As already discussed in relation to the Fabry-Perot fringes, there are ways to overcome these reflections and losses. Here, however, we focus on the properties more specific to the MZI operating under self-collimation conditions, thus leaving this type of improvements for the next phase. The hot spot is also seen at the interface between the PhC and OUT2, but it is much weaker compared to those at IN1 and OUT1, in agreement with the unidirectional output behaviour on collimation. For the off-collimation case of Fig. 6(b), vertical outcoupling at these three positions is present as well, but the hot spot at the interface with OUT2 is much stronger now, in agreement with the loss of unidirectionality. In addition, new spots have appeared at the beam splitters and mirrors, indicating that there is a mismatch of incoming and outgoing in-plane modes at these elements, resulting in vertical out-coupling.

Qualitatively, these far-field observations imply that losses within our PhC MZI operating in the self-collimation mode is negligible compared to waveguide/ $\mathrm{PhC}$ coupling losses. The observation that the vertical out-coupling on collimation is much weaker than off collimation is confirmed by the 3D FDTD simulation of this effect shown in Fig. 4 (green dashed curve). In this simulation, a sensor of $22 \times 22 \mu \mathrm{m}^{2}$ was put centre-to-centre $0.5 \mu \mathrm{m}$ above and in parallel with the MZI plane, to measure the vertically out-coupled radiation. Here a clear dip is seen at $\lambda_{c}$ in an otherwise rather flat curve. The maximum of about 0.06 for this flat curve for the as-designed MZI indicates that other contributions may be involved to account for the complete optical loss. These contributions could be in-plane scattering loss, out-of-plane loss into air with large exit angles (not detected by the parallel sensor in the simulation) or leakage to the $\mathrm{SiO}_{2}$ layer.

\section{Outlook and conclusion}

Our manipulation of self-collimated beams in a PhC to form a compact MZI with a unidirectional output behaviour calls for further research in the direction of making the output of the MZI tunable. To this end one may think of exploiting the sensitivity of the beamsplitter properties to changes of the beam-splitter geometry or its dielectric configuration. In particular, ideas derived from nanoelectromechanical systems (NEMS) may be applied here. 
Active switching can be achieved by introducing a sufficient phase shift locally at the beam splitter BS2, for instance, inserting high refractive index dielectric rods into the air holes [24]. In this way, PhC MZI modulators can have a very small footprint because, unlike in conventional waveguide based modulators, sufficient phase shifts can be achieved without the need of long interferometer arms. NEMS can be operated up to the GHz range [25] and have been combined already with $\mathrm{PhCs}$ to obtain tunability of their transmission [26]. The $\mathrm{PhC}$ MZI presented here is also promising for micromechanical sensing and lab-on-a-chip applications because the phase shifts, induced either locally at a beam splitter or along an interferometer arm, can be monitored as a change of differential output.

In conclusion, we have combined a silicon photonic crystal operating in the selfcollimation mode of infrared light with integrated beam splitters and mirrors, to realize a compact monolithic Mach-Zehnder interferometer (MZI). The resulting MZI has high structural quality and as a result shows a pronounced unidirectionality, as measured with inplane transmission spectroscopy. Further, the self-collimated beams in the $\mathrm{PhC}$ and the unidirectionality have been clearly observed with spatial measurements of the near and far field of the MZI, while simulations have been used to support interpretation of the data. Our results call for development of tuning the MZI output.

\section{Acknowledgment}

This work is supported by NanoNed, a technology program of the Dutch Ministry of Economic Affairs via the foundation STW. 